PROCEEDINGS OF THE

AMERICAN MATHEMATICAL SOCIETY

Volume 125, Number 4, April 1997, Pages 951-960

S 0002-9939(97)03662-9

\title{
ON INVARIANTS DUAL TO THE BASS NUMBERS
}

\author{
EDGAR ENOCHS AND JINZHONG XU \\ (Communicated by Wolmer V. Vasconcelos)
}

\begin{abstract}
Let $R$ be a commutative Noetherian ring, and let $M$ be an $R$ module. In earlier papers by Bass (1963) and Roberts (1980) the Bass numbers $\mu_{i}(p, M)$ were defined for all primes $p$ and all integers $i \geq 0$ by use of the minimal injective resolution of $M$. It is well known that $\mu_{i}(p, M)=$ $\operatorname{dim}_{k(p)} \operatorname{Ext}_{R_{p}}^{i}\left(k(p), M_{p}\right)$. On the other hand, if $M$ is finitely generated, the Betti numbers $\beta_{i}(p, M)$ are defined by the minimal free resolution of $M_{p}$ over the local ring $R_{p}$. In an earlier paper of the second author (1995), using the flat covers of modules, the invariants $\pi_{i}(p, M)$ were defined by the minimal flat resolution of $M$ over Gorenstein rings. The invariants $\pi_{i}(p, M)$ were shown to be somehow dual to the Bass numbers. In this paper, we use homologies to compute these invariants and show that

$$
\pi_{i}(p, M)=\operatorname{dim}_{k(p)} \operatorname{Tor}_{i}^{R_{p}}\left(k(p), \operatorname{Hom}_{R}\left(R_{p}, M\right)\right)
$$

for any cotorsion module $M$. Comparing this with the computation of the Bass numbers, we see that Ext is replaced by Tor and the localization $M_{p}$ is replaced by $\operatorname{Hom}_{R}\left(R_{p}, M\right)$ (which was called the colocalization of $M$ at the prime ideal $p$ by Melkersson and Schenzel).
\end{abstract}

\section{Preliminaries}

Throughout this paper, all rings are commutative Noetherian with identities and all modules are unitary.

We first briefly recall the invariants $\pi_{i}(p, M)$ defined in [10].

By the terminology of Enochs in [4], a linear map $\varphi: F \rightarrow M$ with $F$ flat is called a flat precover of $M$ if $\operatorname{Hom}_{R}(G, F) \rightarrow \operatorname{Hom}_{R}(G, M) \rightarrow 0$ is exact for all flat modules $G$. If furthermore any linear map $f: F \rightarrow F$ such that $f \varphi=\varphi$ is an automorphism of $F$, then $\varphi: F \rightarrow M$ is called a flat cover of $M$. It was proved in [4] that if $M$ has a flat cover, it is unique up to isomorphism. Also, if $M$ has a flat precover $F$, then $M$ has a flat cover which is a direct summand of $F$.

A module $C$ is called cotorsion if $\operatorname{Ext}_{R}^{1}(F, C)=0$ for all flat modules $F$. In the definition of flat covers, if we replace all flat modules by cotorsion modules and reverse the directions of all mappings, we just have the definition of cotorsion envelopes of modules. Covers and envelopes were called minimal approximations by M. Auslander in $[1,2]$.

Received by the editors February 22, 1995 and, in revised form, August 16, 1995.

1991 Mathematics Subject Classification. Primary 13C11, 13 E05.

Key words and phrases. Bass numbers, minimal flat resolutions, cotorsion modules.

(C)1997 American Mathematical Society 
Note that for any injective module $E$, by Matlis' theorem [7], $E$ is a direct sum of indecomposable injective modules such that each of them is in the form $E(R / p)$ for some prime ideal $p$. In general we do not have a similar result for any flat module. But if $F$ is flat and cotorsion, then it was proved in [5, Theorem] that $F$ is uniquely a product $\prod T_{p}$. Here $T_{p}$ is the completion of a free $R_{p}$-module with respect to the $p_{p}$-adic topology. Also note that a flat cover of a cotorsion module is flat and cotorsion, and the kernel of a flat cover $F \rightarrow M$ is cotorsion [5, Lemma 2.2]. Therefore we have the following definitions.

Definition 1.1. Let $R$ be commutative Noetherian, and let $M$ be any $R$-module. A minimal flat resolution of $M$ is an exact sequence

$$
\cdots \rightarrow F_{i} \stackrel{d_{i}}{\rightarrow} F_{i-1} \rightarrow \cdots \rightarrow F_{0} \rightarrow M \rightarrow 0
$$

such that for each $i, F_{i}$ is a flat cover of $\operatorname{im}\left(d_{i}\right)$.

Note that for $i \geq 1, F_{i}$ is flat and cotorsion, and then it is a product of such $T_{p}$. For $i=0, F_{0}$ is not cotorsion in general. But we take its cotorsion envelope (or equivalently pure injective envelope). The pure injective envelope $P E\left(F_{0}\right)$ of $F_{0}$ is flat and cotorsion $\left[6\right.$, p. 352]. Hence, $P E\left(F_{0}\right)$ is a product of $T_{p}$.

Definition 1.2. Assume $M$ has a minimal flat resolution as $(*)$. For $i \geq 1$, $\pi_{i}(p, M)$ is defined to be the cardinality of the base of a free $R_{p}$-module whose completion is $T_{p}$ in the product $F_{i}=\prod T_{q}$. For $i=0, \pi_{0}(p, M)$ is defined similarly by using the pure injective envelope $P E\left(F_{0}\right)$ instead of $F_{0}$ itself.

We note that the $\pi_{i}(p, M)$ are homologically independent if $M$ admits a minimal flat resolution. We call the $\pi_{i}(p, M)$ the dual Bass numbers. In [11, Theorem 2.6], it was proved that every module over a commutative Noetherian ring of finite Krull dimension has a flat cover, and so every module over such a ring admits a minimal flat resolution. Thus, the invariants $\pi_{i}(p, M)$ are well-defined for all modules over coordinate rings of affine algebraic varieties.

\section{THE MAIN RESULTS}

We will see that the computation of $\pi_{i}(p, M)$ for any module $M$ can be reduced to the computation for a cotorsion module.

Theorem 2.1. Let $R$ be commutative Noetherian, and let $M$ be a $R$-module. If $M$ has a flat cover, then $M$ has a cotorsion envelope, denoted $C(M)$. Furthermore, if $M$ admits a minimal flat resolution, so does $C(M)$, and

$$
\pi_{i}(p, M)=\pi_{i}(p, C(M))
$$

for all $i \geq 0$. 
Proof. Let $\sigma_{1}: F \rightarrow M$ be the flat cover of $M$. Consider the following pushout diagram:

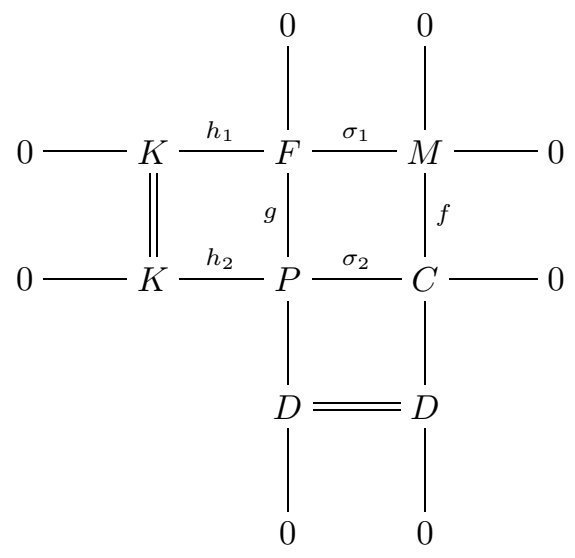

Here, $g: F \rightarrow P$ is a pure injective envelope of $F$. It is flat because $R$ is Noetherian. $C$ is a pushout of $F \rightarrow M$ and $F \rightarrow P$. Since for any cotorsion module $K$, by the definition of cotorsion modules it is easy to argue that $\operatorname{Ext}_{R}^{i}(F, K)=0$ for all $i \geq 1$ whenever $F$ is flat. Then we have that $C$ is cotorsion because both $K$ and $P$ are cotorsion. We note that $D$ is flat $[6$, p. 352]. In the first place, we show that $f: M \rightarrow C$ is a cotorsion preenvelope of $M$. Let $q: P \rightarrow D$, and $p: C \rightarrow D$.

For any linear map $f^{\prime}: M \rightarrow C^{\prime}$ with $C^{\prime}$ cotorsion, we have the diagram

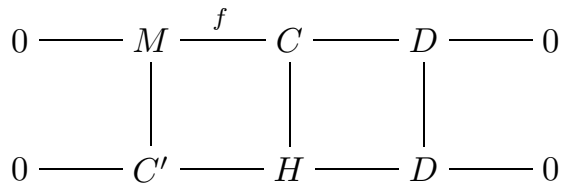

with exact row and columns. Since $D$ is flat and $C^{\prime}$ is cotorsion, the bottom row is split. So we can find a linear map from $C$ to $C^{\prime}$ making the diagram commutative.

Now, we show that the following diagram

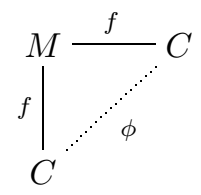

can be completed only by automorphisms of $C$. Suppose $f=\phi f$, we have to prove that $\phi$ is an automorphism of $C$. Consider the diagram:

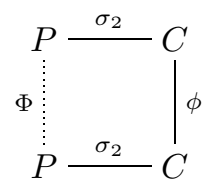

Since $K$ is cotorsion and $P$ is flat, there is a linear map $\Phi: P \rightarrow P$ such that $\sigma_{2} \Phi=\phi \sigma_{2}$. We first show that $\Phi$ is an automorphism of $P$.

Note that $q \Phi g=p \sigma_{2} \Phi g=p \phi \sigma_{2} g=p \phi f \sigma_{1}=p f \sigma_{1}=0$. Then we have $\Phi g: F \rightarrow$ $\operatorname{ker}(q)=g(F)$, and we get a linear map $\alpha=g^{-1} \Phi g: F \rightarrow g(F) \rightarrow F$. 
On the other hand, we have that $f \sigma_{1} g^{-1} \Phi g=\sigma_{2} g g^{-1} \Phi g=\sigma_{2} \Phi g=\phi \sigma_{2} g=$ $\phi f \sigma_{1}=f \sigma_{1}$, or $f\left(\sigma_{1} \alpha-\sigma_{1}\right)=0$. But this implies that $\sigma_{1} \alpha=\sigma_{1}$ because $f$ is an injection.

Consider the diagram

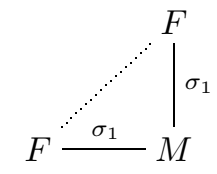

It can be completed only by automorphisms of $F$ because $F$ is a flat cover of $M$. It is clear that $\alpha=g^{-1} \Phi g$ makes the diagram commutative, so it follows that $\alpha$ is an automorphism of $F$ and $g \alpha=\Phi g$.

Next we consider the following diagram

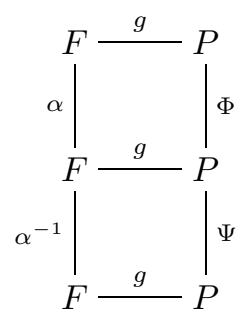

Here $g \alpha=\Phi g$ and $g \alpha^{-1}=\Psi g$. Hence we get that $g=\Phi \Psi g$ and $g=\Psi \Phi g$. These imply that both $\Phi \Psi$ and $\Psi \Phi$ are automorphisms of $P$ because $g: F \rightarrow P$ is a pure injective envelope of $F$. Consequently, $\Phi$ is an automorphism of $P$.

By $\sigma_{2} \Phi=\phi \sigma_{2}$, we know that $\phi$ is onto. It remains to show that $\phi$ is also injective. In order to do this, we make the following calculations.

For any $x \in \operatorname{ker}(\phi), x=\sigma_{2}(y)$ for some $y \in P$. We claim that $x=0$. Note that $0=\phi(x)=\phi \sigma_{2}(y)=\sigma_{2} \Phi(y)$, so then $\Phi(y) \in \operatorname{ker}\left(\sigma_{2}\right)$. But $\Phi(y)=h_{2}(k)$ for some $k \in K$. Consider $h_{1}(k) \in F$, since $\alpha=g^{-1} \Phi g$ is an automorphism of $F$, there is a $w \in F$ such that $h_{1}(k)=\alpha(w)$. Now note that $0=\sigma_{1} h_{1}(k)=\sigma_{1} \alpha(w)=\sigma_{1}(w)$. This means that $w \in \operatorname{ker}\left(\sigma_{1}\right)=h_{1}(K)$. But then, $\Phi(y)=h_{2}(k)=g h_{1}(k)=$ $g \alpha(w)=g g^{-1} \Phi g(w)=\Phi g(w)$, and so implies $y=g(w)$. Therefore, $x=\sigma_{2}(y)=$ $\sigma_{2} g(w)=f \sigma_{1}(w)=0$. Thus we have proved that $\phi$ is an automorphism and $f: M \rightarrow C$ is a cotorsion envelope of $M$, denoted $C(M)$.

Finally, by the proof above, it is not hard to see that $\sigma_{2}: P \rightarrow C$ is a flat cover of $C$ and $P$ is cotorsion flat itself. Then by the definition of the invariants we get $\pi_{i}(p, M)=\pi_{i}(p, C(M))$ for all $i \geq 0$.

Theorem 2.2. Let $R$ be commutative Noetherian, and let $M$ be a cotorsion $R$ module which admits a minimal flat resolution. Then

$$
\pi_{i}(p, M)=\operatorname{dim}_{k(p)} \operatorname{Tor}_{i}^{R_{p}}\left(k(p), \operatorname{Hom}_{R}\left(R_{p}, M\right)\right)
$$

for all prime ideals $p$ and all $i \geq 1$ (here $k(p)=(R / p)_{p}$ is the field of fractions at $p)$.

Note that $\operatorname{Hom}_{R}\left(R_{p}, M\right)$ is called the colocalization of $M$ at $p$ in [8]. Before the proof we need several lemmas.

Lemma 2.3. Let $\varphi: F \rightarrow M$ be a flat cover of $M$ and $F=F_{1} \oplus F_{2}$. Then the restriction map $\left.\varphi\right|_{F_{1}}$ cannot be factored through the restriction $\left.\varphi\right|_{F_{2}}$ unless $F_{1}=0$. 
Proof. Let $f: F_{1} \rightarrow F_{2}$ be such that $\left(\left.\varphi\right|_{F_{2}}\right) \circ f=\left.\varphi\right|_{F_{1}}$. Then the diagram

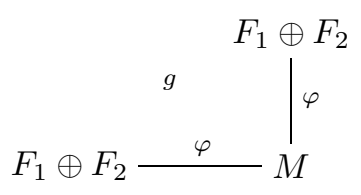

is commutative. Here $g: F_{1} \oplus F_{2} \rightarrow F_{1} \oplus F_{2}$ is defined by $g(a+b)=f(a)+b$ for any $x=a+b$ in $F_{1} \oplus F_{2}, a \in F_{1}, b \in F_{2}$. Note that the image of $g$ is in $F_{2}$. By the definition of a flat cover, $g$ must be an automorphism. This is impossible unless $F_{1}$ is zero.

Lemma 2.4. Let $R$ be commutative Noetherian, and let $p$ be a prime ideal in $R$. Let $\varphi: \widehat{R_{p}} \rightarrow F$ be $R_{p}$-linear with an $R_{p}$-flat module $F$, and $\sigma: F \rightarrow(R / p)_{p}$ be an $R_{p}$-linear map. If the composition $\sigma \circ \varphi$ is nonzero, then $\varphi$ is an injection and its image is a direct summand of $F$.

Proof. Note that any nonzero $R_{p}$-linear map $\bar{\varphi}: \widehat{R_{p}} \rightarrow(R / p)_{p}$ is a flat cover of $k(p)=(R / p)_{p}$ as $R_{p}$-module [5, Example, p. 181]. Then $\bar{\varphi}=\sigma \circ \varphi: \widehat{R_{p}} \rightarrow(R / p)_{p}$ is a flat cover. Since $F$ is flat, there is a linear map $\alpha: F \rightarrow \widehat{R_{p}}$ such that $\sigma=\bar{\varphi} \circ \alpha$ by the definition of flat covers. Easily, we have $\bar{\varphi}=\sigma \circ \varphi=\bar{\varphi} \circ \alpha \circ \varphi$. Hence $\alpha \circ \varphi$ is an automorphism of $\widehat{R_{p}}$. Therefore, it follows that $\varphi$ is an injection and its image is a direct summand of $F$.

Lemma 2.5. Let $R$ be commutative Noetherian, and let $F=\prod T_{q}$ be a flat cotorsion $R$-module. If $L \subset F$ is a direct summand of $F$ isomorphic to $\widehat{R_{p}}$, then $\prod_{q \subsetneq p} T_{q} \cap L=0$ and $\prod_{q \subsetneq p} T_{q} \oplus L$ is a direct summand of $F$.

Proof. We have $F=\prod_{q \subseteq p} T_{q} \oplus \prod_{q \nsubseteq p} T_{q}=L \oplus X$ with $L \cong \widehat{R_{p}}$. Let $Z=\prod_{q \nsubseteq p} T_{q}$. We claim that $L \subseteq \prod_{q \subseteq p} T_{q}$. Otherwise, let

$$
\alpha: F \rightarrow \prod_{q \nsubseteq p} T_{q}
$$

be the projection. Then the restriction to $L,\left.\alpha\right|_{L}$, is not zero, and the image is contained in $Z$. Easily, $\operatorname{Hom}\left(\widehat{R_{p}}, \alpha(L)\right)$ is not zero. But $\operatorname{Hom}\left(\widehat{R_{p}}, T_{q}\right)=0$ for any $q$ which is not contained in $p$ by [6, Lemma 1.3]. This implies that $\operatorname{Hom}\left(\widehat{R_{p}}, Z\right)=0$, and then $\operatorname{Hom}\left(\widehat{R_{p}}, \alpha(L)\right)=0$. This is a contradiction.

Now we have $\prod_{q \subseteq p} T_{q}=\prod_{q \subseteq p} T_{q} \cap F=L \oplus X \cap \prod_{q \subseteq p} T_{q}$.

We further claim that $Y=\prod_{q \subseteq p} T_{q}$ is contained in $X$. Otherwise, let $h: F=$ $L \oplus X \rightarrow L$ be the projection. Then the image of the restriction on $Y, h(Y) \neq 0$. But by [6, Lemma 1.3], $\operatorname{Hom}\left(Y, \widehat{R_{p}}\right)=0$, and then $\operatorname{Hom}\left(h(Y), \widehat{R_{p}}\right)=0$. But note that $h(Y)$ is a submodule of $L$ which is isomorphic to $\widehat{R_{p}}$. Then $\operatorname{Hom}(h(Y), L) \neq 0$. This is a contradiction. Therefore, we have

$$
\begin{gathered}
\prod_{q \subseteq p} T_{q}=L \oplus \prod_{q \subsetneq p} T_{q} \oplus X \cap T_{p}, \text { and } \\
F=L \oplus \prod_{q \subsetneq p} T_{q} \oplus X \cap T_{p} \oplus \prod_{q \nsubseteq p} T_{q} .
\end{gathered}
$$


Lemma 2.6. Let $R$ be commutative Noetherian, and let $C$ be a cotorsion $R$-module. Let $\varphi: F=\prod T_{q} \rightarrow C$ be a flat precover. Then $\varphi$ is a flat cover if and only if for any prime ideal $p$ and any direct summand $L \subset F$ of $F$ isomorphic to $\widehat{R_{p}}$, the diagram

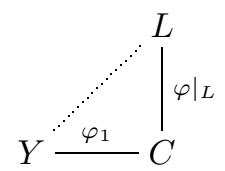

cannot be completed to a commutative one. Here, $Y=\prod_{q \subsetneq p} T_{q}$ and $\varphi_{1}$ is the restriction to $Y$.

Proof. By Lemma 2.5, $F=L \oplus X=L \oplus Y \oplus Z$. Suppose $\varphi$ is a flat cover of $C$. If the diagram can be completed commutatively, then we have a contradiction by Lemma 2.3.

Conversely, suppose $\varphi$ has the given property. Let $K=\operatorname{ker}(\varphi)$. If $\varphi$ is not a flat cover, then $K$ contains a nonzero direct summand $H$ of $F$ by [5, Lemma 1.1]. Since $H$ is flat and cotorsion, it will have a direct summand $L$ isomorphic to $\widehat{R_{p}}$ for some prime ideal $p$. Then the restriction $\left.\varphi\right|_{L}=0$. Easily the corresponding diagram can be completed commutatively by the zero map. This contradicts the condition.

Theorem 2.7. Let $R$ be commutative Noetherian, and let $C$ be cotorsion. Let $\varphi: F \rightarrow C$ be a flat cover of $C$. Then if $S \subset R$ is multiplicative,

$$
\operatorname{Hom}\left(S^{-1} R, F\right) \rightarrow \operatorname{Hom}\left(S^{-1} R, C\right)
$$

is a flat cover.

Proof. Using the natural isomorphisms

$$
\operatorname{Hom}\left(G, \operatorname{Hom}\left(S^{-1} R, F\right)\right) \cong \operatorname{Hom}\left(G \otimes S^{-1} R, F\right)
$$

and

$$
\operatorname{Hom}\left(G, \operatorname{Hom}\left(S^{-1} R, C\right)\right) \cong \operatorname{Hom}\left(G \otimes S^{-1} R, C\right)
$$

and the fact that $G \otimes S^{-1} R$ is flat if $G$ is flat, we see that $\operatorname{Hom}\left(S^{-1} R, F\right)$ is a flat precover of $\operatorname{Hom}\left(S^{-1} R, C\right)$. If it is not a flat cover, then its kernel contains a direct summand $L$ isomorphic to $\widehat{R_{p}}$ for some prime ideal $p$ [5, Lemma 1.1 and Theorem].

Since $\operatorname{Hom}\left(S^{-1} R, F\right)$ and $L$ both are $S^{-1} R$-modules, $S^{-1} \operatorname{Hom}\left(S^{-1} R, F\right)=$ $\operatorname{Hom}\left(S^{-1} R, F\right), S^{-1} L=L$ and $S^{-1} \widehat{R_{p}}=\widehat{R_{p}}$. We get that $\widehat{R_{p}} \cong \operatorname{Hom}\left(\widehat{R_{p}}, \widehat{R_{p}}\right)$ is isomorphic to a direct summand of $\operatorname{Hom}\left(\widehat{R_{p}}, \operatorname{Hom}\left(S^{-1} R, F\right)\right)$, and contained in the kernel of

$$
\operatorname{Hom}\left(\widehat{R_{p}}, \operatorname{Hom}\left(S^{-1} R, F\right)\right) \rightarrow \operatorname{Hom}\left(\widehat{R_{p}}, \operatorname{Hom}\left(S^{-1} R, C\right)\right) .
$$

But $\operatorname{Hom}\left(\widehat{R_{p}}, \operatorname{Hom}\left(S^{-1} R, F\right)\right) \cong \operatorname{Hom}\left(S^{-1} \widehat{R_{p}}, F\right) \cong \operatorname{Hom}\left(\widehat{R_{p}}, F\right)$. Likewise for $\operatorname{Hom}\left(\widehat{R_{p}}, \operatorname{Hom}\left(S^{-1} R, C\right)\right)$.

Hence, $\operatorname{Hom}\left(\widehat{R_{p}}, \varphi\right): \operatorname{Hom}\left(\widehat{R_{p}}, F\right) \rightarrow \operatorname{Hom}\left(\widehat{R_{p}}, C\right)$ is such that there is a direct summand $L$ of $\operatorname{Hom}\left(\widehat{R_{p}}, F\right)$ isomorphic to $\widehat{R_{p}}$ and which is in the kernel of $\operatorname{Hom}\left(\widehat{R_{p}}, F\right) \rightarrow \operatorname{Hom}\left(\widehat{R_{p}}, C\right)$. 
Now note that $\operatorname{Hom}\left(\widehat{R_{p}}, T_{q}\right)=0$ for any prime ideal $q$ which is not contained in $p$. Let $F=\prod T_{q}$, and $Y=\prod_{q \subsetneq p} T_{q}$. Then we have that

$$
\operatorname{Hom}\left(\widehat{R_{p}}, F\right)=\operatorname{Hom}\left(\widehat{R_{p}}, \prod_{q \subseteq p} T_{q}\right)=\operatorname{Hom}\left(\widehat{R_{p}}, T_{p}\right) \oplus \operatorname{Hom}\left(\widehat{R_{p}}, Y\right) .
$$

Let $(\sigma, g), \sigma \in \operatorname{Hom}\left(\widehat{R_{p}}, T_{p}\right), g \in \operatorname{Hom}\left(\widehat{R_{p}}, Y\right)$ be a generator of $L$ as an $\widehat{R_{p}}$ module. Then we claim that $\sigma: \widehat{R_{p}} \rightarrow T_{p}$ maps $\widehat{R_{p}}$ isomorphically onto a direct summand of $T_{p}$. To see this, by the proof of Lemma 2.4 we only need to show that $\sigma\left(\widehat{R_{p}}\right) \nsubseteq p T_{p}$. Since $(\sigma, g)$ is a generator of $L$ which is isomorphic to $\widehat{R_{p}}$, it follows that

$$
(\sigma, g) \notin p \operatorname{Hom}\left(\widehat{R_{p}}, T_{p}\right) \oplus p \operatorname{Hom}\left(\widehat{R_{p}}, Y\right) .
$$

But note that $p \operatorname{Hom}\left(\widehat{R_{p}}, T_{p}\right) \cong \operatorname{Hom}\left(\widehat{R_{p}}, p T_{p}\right)$ and $p \operatorname{Hom}\left(\widehat{R_{p}}, Y\right)=\operatorname{Hom}\left(\widehat{R_{p}}, Y\right)$. Hence if we assume $\sigma\left(\widehat{R_{p}}\right) \subset p T_{p}$, then we will have that

$$
\begin{aligned}
(\sigma, g) & \in p\left(\operatorname{Hom}\left(\widehat{R_{p}}, T_{p}\right) \oplus p \operatorname{Hom}\left(\widehat{R_{p}}, Y\right)\right) \\
& =p\left(\operatorname{Hom}\left(\widehat{R_{p}}, T_{p}\right) \oplus \operatorname{Hom}\left(\widehat{R_{p}}, Y\right)\right) .
\end{aligned}
$$

This implies that $(\sigma, g) \in p L$ since $L$ is a direct summand of

$$
\operatorname{Hom}\left(\widehat{R_{p}}, F\right) \cong \operatorname{Hom}\left(\widehat{R_{p}}, T_{p}\right) \oplus \operatorname{Hom}\left(\widehat{R_{p}}, Y\right) \text {. }
$$

Obviously this is impossible since $L \cong \widehat{R_{p}}$. Therefore the claim has been asserted.

Finally, since $(\sigma, g)$ is in the kernel of $\operatorname{Hom}\left(\widehat{R_{p}}, \varphi\right),\left(\left.\varphi\right|_{T_{p}}\right) \circ \sigma+\left(\left.\varphi\right|_{Y}\right) \circ g=0$. But then

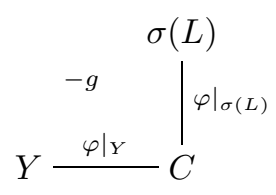

is a commutative diagram. This contradicts the fact that $\varphi: F \rightarrow C$ is a flat cover by Lemma 2.6 .

Proof of Theorem 2.2. Let the following resolution be a minimal flat resolution of $M$ :

$$
\cdots \rightarrow G_{n+1} \rightarrow G_{n} \rightarrow \cdots \rightarrow G_{0} \rightarrow M \rightarrow 0 .
$$

By Theorem 2.7, taking colocalization at $p$, we have a minimal flat resolution of $\operatorname{Hom}\left(R_{p}, M\right)$ as an $R_{p}$-module,

$$
\cdots \rightarrow F_{n+1} \stackrel{\stackrel{\partial}{\rightarrow}}{\rightarrow} F_{n} \rightarrow \cdots \rightarrow F_{0} \rightarrow \operatorname{Hom}\left(R_{p}, M\right) \rightarrow 0,
$$

where $F_{i}=\operatorname{Hom}\left(R_{p}, G_{i}\right)$ for all $i$. Since each $F_{i}$ is cotorsion and flat as $R_{p}$-module, $F_{i}=T_{p} \oplus \prod_{q \subsetneq p} T_{q}$. Hence

$$
k(p) \otimes F_{i}=k(p) \otimes T_{p} \oplus k(p) \otimes \prod_{q \subsetneq p} T_{q}=\frac{T_{p}}{p T_{p}} .
$$


Let $\bar{\partial}$ stand for $k(p) \otimes \partial$. We claim that $\bar{\partial}$ is zero. Suppose $\bar{\partial}$ is not zero. Then $\bar{\partial}(\bar{y}) \neq 0$ for some $y \in F_{n}$. We may assume that $y$ is in $T_{p} \subset F_{n}$. Then we consider the following diagram:

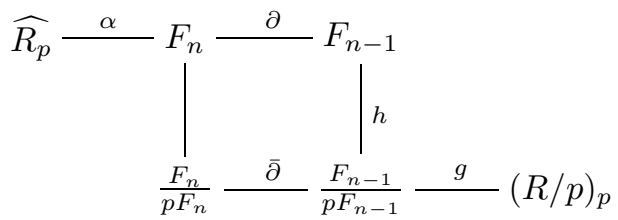

Here, $\alpha: \widehat{R_{p}} \rightarrow F_{n}$ is defined by sending $r$ to $r y$ for every $r \in \widehat{R_{p}}$, and $g$ is a projection such that $g(\bar{\partial}(\bar{y})) \neq 0$. Now let $\varphi=\partial \circ \alpha, \sigma=g \circ h$. Then $\sigma \circ \varphi$ is not zero. By Lemma 2.4, $\varphi=\partial \circ \alpha$ is an injection and its image is a direct summand of $F_{n-1}$. Actually, the image is in the kernel of $F_{n-1} \rightarrow F_{n-2}$. This contradicts the fact that $\operatorname{Hom}\left(R_{p}, *\right)$ is a minimal flat resolution of $\operatorname{Hom}\left(R_{p}, M\right)$ by Theorem 2.7.

Therefore, $\operatorname{dim}_{k(p)} \operatorname{Tor}_{i}^{R_{p}}\left(k(p), \operatorname{Hom}\left(R_{p}, M\right)\right)=\operatorname{dim}_{k(p)} \frac{F_{i}}{p F_{i}}=\operatorname{dim}_{k(p)} \frac{T_{p}}{p T_{p}}$. This is just the cardinality of the base of a free $R_{p}$-module whose completion is $T_{p}$ in the product $F_{i}=\prod T_{q}$.

Example. Let $(R, m)$ be local commutative Noetherian, and let $k(m)=R / m$. Then for any prime ideal $p$ which is not maximal, we have $\operatorname{Hom}\left(R_{p}, k(m)\right)=0$, and so $\pi_{i}(p, k(m))=0$ for all $i$. But for the maximal ideal $m$, we have that

$$
\begin{aligned}
\pi_{i}(m, k(m)) & =\operatorname{dim}_{k(m)} \operatorname{Tor}_{i}^{R_{m}}\left(k(m), \operatorname{Hom}\left(R_{m}, k(m)\right)\right) \\
& =\operatorname{dim}_{k(m)} \operatorname{Tor}_{i}^{R_{m}}(k(m), k(m))
\end{aligned}
$$

are finite for all $i$. In fact, they are equal to the Betti numbers of $k(m)$.

Comparing the dual Bass invariants $\pi_{i}(p, M)$ with the Betti numbers $\beta_{i}(M)$, we found the most important advantage is that $\pi_{i}(p, M)$ can be defined for nonfinitely generated modules. In particular, the $\pi_{i}(p, E(R / m))$ are well defined and wellbehaved when $m$ is a maximal ideal of $R$. In general, we have the following fact.

Proposition 2.8. Let $R$ be commutative Noetherian. Then for any Artinian module $M$, the dual Bass invariants $\pi_{i}(p, M)$ are all well defined, and they can be computed by the formula of Theorem 2.2. Furthermore if $M$ is of finite length, then $\pi_{i}(p, M)=0$ for nonmaximal prime ideal $p$ and all integers $i \geq 0$. For any maximal ideal $m$, all $\pi_{i}(m, M)$ are finite.

Proof. We first show that any Artinian module $M$ is cotorsion, and admits a minimal flat resolution.

Note that $M$ is an essential submodule of $E\left(R / m_{1}\right) \oplus \cdots \oplus E\left(R / m_{t}\right)$ for finitely many maximal ideals $\left\{m_{1}, \ldots, m_{t}\right\}$. Among these maximal ideals we may assume that $m_{1}, \ldots, m_{s}$ are different. Then it is not hard to argue that there is a direct decomposition of $M$ :

$$
M=M_{1} \oplus M_{2} \oplus \cdots \oplus M_{s},
$$

where $M_{i}=\left\{x \in M \mid m_{i}^{l} x=0\right.$ for some integer $\left.l\right\}$. This implies that each $M_{i}$ is Artinian as an $R_{m_{i}}$-module and $\widehat{R}_{m_{i}}$-module. Furthermore, $M_{i}$ is Matlis reflexive as an $\widehat{R}_{m_{i}}$-module, and then it is cotorsion as an $\widehat{R}_{m_{i}}$-module. But it follows that $M_{i}$ is cotorsion as an $R$-module by noting the fact that $M_{i} \otimes_{R} \widehat{R}_{m_{i}} \cong M_{i}$. Therefore $M$ itself is cotorsion as an $R$-module. Also note that each $M_{i}$ has a minimal flat 
resolution as an $R_{m_{i}}$-module since $R_{m_{i}}$ has finite Krull dimension [11, Theorem 2.6]. This shows that $M_{i}$ has minimal flat resolution as an $R$-module [11, Corollary 2.7], and hence so does $M$ itself.

Next we assume $M$ to be of finite length. Note that $\operatorname{Hom}\left(R_{p}, M\right)=0$ for any prime ideal which is not maximal. For every maximal ideal $m$ it is not hard to see that $\operatorname{Hom}\left(R_{m}, M\right)$ has a finite length. Then the conclusion follows by Theorem 2.2. This finishes our proof.

So far we do not know if every cotorsion module admits a minimal flat resolution. But for any $R$-module $M$ and any injective module $R$-module $E, \operatorname{Hom}(M, E)$ is pure injective (so cotorsion), and admits a minimal flat resolution. Moreover we have $\operatorname{Tor}_{i}^{R_{p}}\left(k(p), \operatorname{Hom}_{p}\left(R_{p}, \operatorname{Hom}_{R}(M, E)\right)\right) \cong \operatorname{Hom}_{R}\left(\operatorname{Ext}_{R_{p}}^{i}\left(k(p), M_{p}\right), E\right)$. In particular, we have the following.

Proposition 2.9. Let $R$ be commutative Noetherian. Then for any prime ideal $p, R_{p}$ is Cohen-Macaulay if and only if $\pi_{i}(p, E(R / p))=0$ for $i<h t(p) . R_{p}$ is Gorenstein if and only if $\pi_{i}(p, E(R / p))=0$ for $i<h t(p)$ and $\pi_{h t(p)}(p, E(R / p))=1$.

Proof. By the above it is easy to see that $\pi_{i}(p, E(R / p))$ is equal to $\mu_{i}(p, R)$. Then the conclusion follows by (3.7) and (4.1) of [3].

We conclude this paper with the following notes. For a module $M$ over a commutative Noetherian ring $R$, if $M$ has finite flat dimension $d \geq 0$, then $M$ has a minimal flat resolution (see Thm. $2.3[11]$ ). Hence $d$ is just the largest integer such that $\pi_{d}(p, M) \neq 0$ for some prime ideal $p$. In general, if $d$ is the smallest integer such that $\pi_{d}(p, M)=0$ for all prime $p$, then the flat dimension of $M$ is less than or equal to $d-1$.

Now assume $(R, m)$ to be a commutative Noetherian local ring. Let $A$ be a finitely generated $R$-module. Then we have a minimal free resolution of $A$ with the $i$ th term a finite rank free $R$-module. Let $\widehat{R}$ be the completion of $R$ with respect to $m$-adic topology. By applying the tensor functor $-\otimes_{R} \widehat{R}$ to the free resolution of $A$, we get a free resolution of $\widehat{A}=A \otimes_{R} \widehat{R}$ as an $\widehat{R}$-module. Furthermore it can be easily seen that this resolution is in fact a minimal flat resolution of $\widehat{A}$ as $\widehat{R}$-module. Therefore, we have $\beta_{i}^{R}(A)=\beta_{i}^{\widehat{R}}(\widehat{A})=\pi_{i}^{\widehat{R}}(\widehat{m}, \widehat{A})$.

\section{ACKNOWLEDGEMENT}

The authors of this paper want to express their gratitude to the referee for his careful reading and good suggestions.

\section{REFERENCES}

1. M. Auslander and I. Reiten, Applications of contravariantly finite subcategories, Adv. Math. 86 (1991), 111-152. MR 92e:16009

2. M. Auslander and R. Buchweitz, The homological theory of maximal Cohen-Macaulay approximations, Soc. Math. de France, Memoire 38 (1989), 5-37. MR 91h:13010

3. H. Bass, On the ubiquity of Gorenstein rings, Math. Z. 82 (1963), 8-28. MR 27:3669

4. E. Enochs, Injective and flat covers, envelopes and resolvents, Israel J. of Math. 39 (1981), 33-38. MR 83a:16031

5. _ Flat covers and flat cotorsion modules, Proc. Amer. Math. Soc. 92 (1984), 179-184. MR 85j: 13016

6. _ Minimal pure injective resolutions of flat modules, J. of Algebra 105 (2) (1987), 351-364. MR 88f:13001 
7. E. Matlis, Injective modules over Noetherian rings, Pacific J. Math. 8 (1958), 511-528. MR 20:5800

8. Leif Melkersson and Peter Schenzel, The co-localization of an Artinian module, Proc. Edinburgh Math. Soc. (2) 38 (1995), 121-131. MR 96a:13020

9. P. Roberts, Homological invariants of modules over commutative rings, Les Presses de l'Univ. de Montreal, 1980. MR 82j:13020

10. Jinzhong Xu, Minimal injective and flat resolutions of modules over Gorenstein rings, J. of Algebra 175 (1995), 451-477. MR 96h:13025

11. _ The existence of flat covers over Noetherian rings of finite Krull dimension, Proc. Amer. Math. Soc. 123 (1) (1995), 27-32. MR 95c:16004

Department of Mathematics, University of Kentucky, Lexington, Kentucky 40506 